\title{
Digital Design and Nonlinear Simulation for Additive Manufacturing of Soft Lattice Structures
}

\author{
O. Weeger ${ }^{1}$, N. Boddeti ${ }^{1}$, S.-K. Yeung ${ }^{2}$, S. Kaijima ${ }^{3}$, M. L. Dunn ${ }^{4}$ \\ ${ }^{1}$ SUTD Digital Manufacturing and Design Centre, Singapore University of Technology and Design, \\ 8 Somapah Road, Singapore 487372, Singapore \\ ${ }^{2}$ Hong Kong University of Science and Technology, Division of Integrative Systems and Design, \\ Room 6530, 6/F, School of Engineering, Clear Water Bay, Kowloon, Hong Kong \\ ${ }^{3}$ Harvard University, Graduate School of Design, \\ 48 Quincy St, Cambridge, MA 02138, USA \\ ${ }^{4}$ University of Colorado Denver, College of Engineering and Applied Science, \\ P.O. Box 173364, Campus Box 104, Denver, CO 80217-3364, USA \\ oliver_weeger@sutd.edu.sg, gowri_boddeti@sutd.edu.sg, \\ saikit@ust.hk,skaijima@gsd.harvard.edu,martin.dunn@ucdenver.edu
}

\section{Abstract}

Lattice structures are frequently found in nature and engineering due to their myriad attractive properties, with applications ranging from molecular to architectural scales. Lattices have also become a key concept in additive manufacturing, which enables precise fabrication of complex lattices that would not be possible otherwise. While design and simulation tools for stiff lattices are common, here we present a digital design and nonlinear simulation approach for additive manufacturing of soft lattices structures subject to large deformations and instabilities, for which applications in soft robotics, healthcare, personal protection, energy absorption, fashion and design are rapidly emerging. Our framework enables design of soft lattices with curved members conforming to freeform geometries, and with variable, gradually changing member thickness and material, allowing the local control of stiffness. We model the lattice members as 3D curved rods and using a splinebased isogeometric method that allows the efficient simulation of nonlinear, large deformation behavior of these structures directly from the CAD geometries. Furthermore, we enhance the formulation with a new joint stiffening approach, which is based on parameters derived from the actual node geometries. Simulation results are verified against experiments with soft lattices realized by PolyJet multi-material polymer 3D printing, highlighting the potential for simulation-driven, digital design and application of non-uniform and curved soft lattice structures. 


\section{Keywords}

Lattices; digital design; multi-material 3D printing; soft structures; functionally graded structures; nonlinear rods

\section{Introduction}

Lattice structures and other types of meta-materials have become an important application of additive manufacturing (AM) methods, since AM processes enable the fabrication of tailored materials with complex microstructures, as well as freeform geometric shapes at the macroscale. Inspiration for such micro-structured materials is often taken from nature, where cellular materials have emerged due to their myriad attractive properties, such as high stiffness-to-density ratios, and the ability to realize spatially-varying material behavior, i.e., stiffness, anisotropy, and density.

In engineering applications of lattice structures, i.e., cellular materials with truss-like microstructures, the focus has largely been on lightweight, load-carrying parts, e.g., for the aerospace industry or medical implants, which can be precisely fabricated through metal 3D printing processes such as selective laser melting (SLM) and electron beam melting (EBM) [1], or polymers via stereolithography (SLA) and selective laser sintering (SLS). The mechanical performance of these stiff lattice structures is characterized by small deformations, i.e., linear elastic and plastic behavior, as well as fracture and failure. Most of the modelling and simulation research, as well as commercial computer-aided design (CAD) tools, focus on these stiff lattice structures $[2,3,4,5,6,7]$.

In contrast, soft lattice structures are characterized by the ability to achieve elastic large deformation behavior through geometric and/or material nonlinearities [8], exploit mechanical instabilities [9, 10], tailor spatially varying material properties, and conform to freeform design spaces through morphed and curved strut architectures. These properties are highly desirable for many types of applications, such as reusable energy absorbing devices based on nonlinear elastic rather than plastic deformations, vibration mitigation through curved ligaments [11], tailored energy absorption response through functional grading of material properties or hierarchical microstructures [12], tissue-like medical implants, soft robotic actuators and devices [13], 4D printing $[14,15]$, or structures with complex constitutive behavior such as negative Poisson's ratio effects [16, $17,18]$.

Soft lattice structures are still an emerging direction of scientific research and practical application. They can be realized through 3D printing elastomeric materials, e.g., with high-resolution material jetting methods such as PolyJet, SLA, digital light processing (DLP), where printing of extremely stretchable materials was recently demonstrated [19], Continuous Liquid Interface Production (CLIP) technology, which is already being used commercially for a lattice-type shoe sole [20], or 3D printing of molds followed by injection of an elastomeric 
material [8]. An important aspect for the feasibility of AM of lattice structures, or in general any type of metamaterial, is the requirement of support materials and structures for the 3D printing process of overhanging structures. Due to the geometric complexity of lattices, mechanical removal of internal supports is in general not possible and consequently, fabrication of lattice structures has so far been limited to certain types of unit cell designs and manufacturing processes that do not require support, e.g. DLP, or powder bed supports in SLS. With the recent development of soluble support materials for PolyJet 3D printing [21], it has become feasible to manufacture lattices with arbitrary unit cells and morphed topologies, and monolithically fabricate hybrid assemblies of lattices with shells and solids even from multiple materials.

This work presents a computational design for additive manufacturing framework for soft lattice structures, that addresses the complete workflow from CAD, to nonlinear mechanical simulation, to re-design, and to manufacture using PolyJet multi-material 3D printing. Design capabilities include the ability to specify global parameters such as 3D freeform design domains and arbitrary unit cell designs that are used to generate spacefilling conformal lattice grids with curved struts, see Figure 1 (a), as well as tailored, local grading of lattice geometry and elasticity through spatially variable strut diameter distributions, see Figure 1 (b). Similarly, spatially variable strut material properties, i.e., Young's modulus, could be defined and realized through voxellevel PolyJet printing [22].

An important aspect of our framework is the prediction of the mechanical deformation behavior of soft 3D lattices, see Figure 1 (c), which is characterized by nonlinear elastic deformations, large strains and instabilities such as buckling. A common approach to the analysis and optimization of metamaterials and lattices is multiscale simulation through homogenization of unit cell behavior [23, 24, 25, 26]. However, due to the functional grading and curved topology, which lead to non-periodicity of the microstructure, as well as the nonlinearity of a soft lattice, common homogenization approaches cannot be applied and the lattice needs to be simulated at full scale. In addition, nonlinear simulation based on continuum mechanics and 3D finite elements is not feasible due to the huge computational effort involved and linear modelling with 3D truss elements will not capture the nonlinear behavior of a soft lattice.

A main contribution of our work is thus a novel approach that balances accuracy with computational efficiency by modeling the lattice struts as nonlinear curved 3D beams. Furthermore, we develop a new joint-stiffening approach similar to the one presented in $[23,28]$, which takes into account, in an approximate manner, the impact of the 3D geometry of lattices nodes on the overall deformation of lattice structures by increasing the diameters of struts at joints. We employ an isogeometric collocation method [27] for the numerical discretization of the beam representation of a lattice structure, which enables the integration with the CAD environment by a 
common NURBS description of centerline curves and the modeling of varying strut diameters through NURBS parameterizations.

An overview of our computational framework for the design, simulation and fabrication of soft lattices is shown in Figure 1 and its main components, the computer-aided design of the lattice structure in terms of its global parameters such as design space, unit cell type and conformity (a), its local, graded and spatially varying material properties or strut diameters (b), its computational simulation and performance evaluation for given boundary conditions (c), which serves as a basis for iterative re-design or automated design optimization processes from (c) back to (a) or (b), as well as the manufacture using PolyJet 3D printing (d-f), are described in full detail in the Methods section. The framework and the accuracy of the mechanical simulation approach are evaluated in the Results and discussion section and application-oriented design examples are demonstrated and discussed, before the Conclusion of the paper.

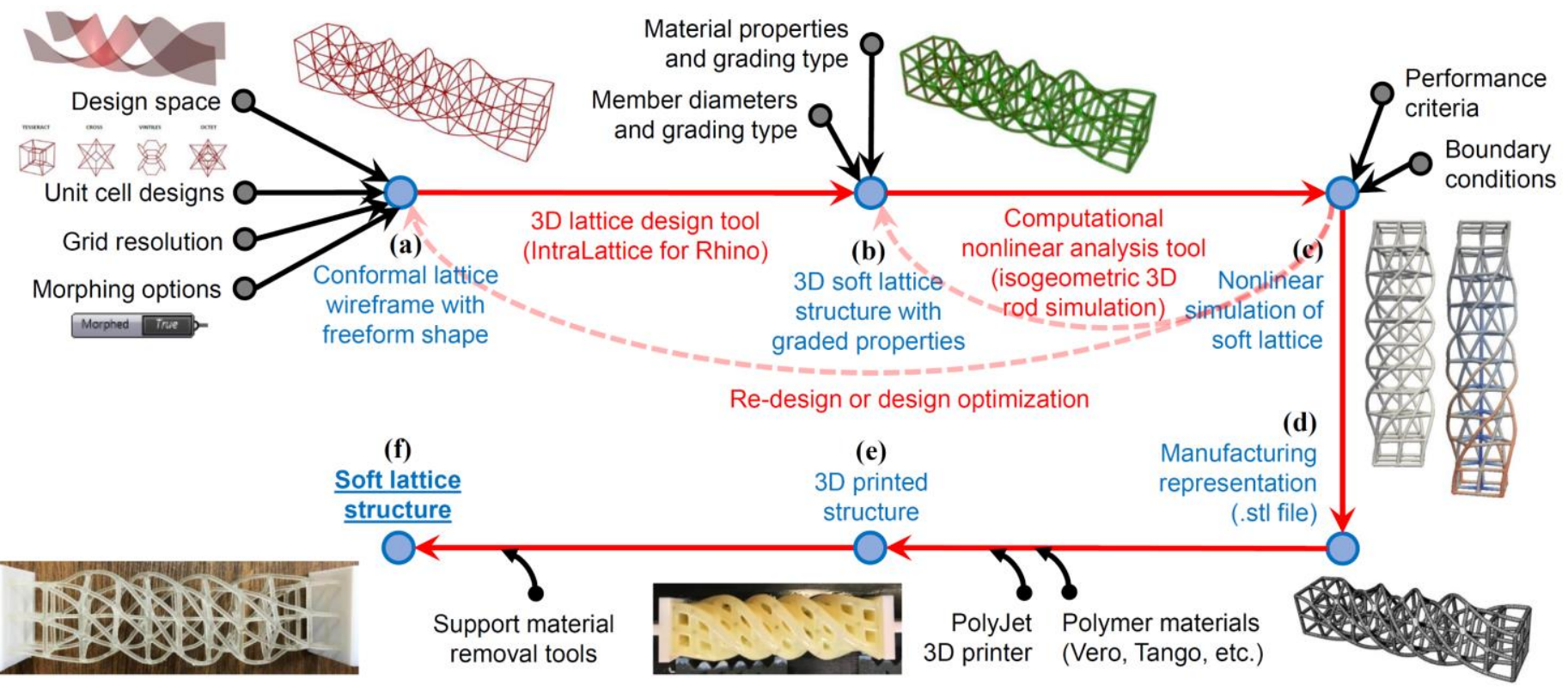

Figure 1. Overview of the digital design and manufacturing framework for soft lattice structures. The main steps are (a) the generation of a conformal wireframe structure, (b) its solidification into a graded 3D lattice structure, (c) nonlinear mechanical simulation of the lattice, $(d+e)$ 3D printing using a PolyJet 3D printer with soluble support material, and $(f)$ support material removal, i.e. dissolving, to obtain the final artefact. 


\section{Methods}

\subsection{Computer-aided design of soft lattice structures}

\subsubsection{Computer-aided design framework}

We developed a computer-aided design tool for the generation of lattice structures with conformal freeform geometries and spatially variable, graded strut diameters, which is based on two major steps: the generation of the initial lattice wireframe and its solidification. The design approach was implemented in the 3D modeling software Rhinoceros ${ }^{\circledR}$ (also Rhino ${ }^{\circledR}$ or Rhino $3 D{ }^{\circledR}$ ) using the algorithmic design environment Grasshopper ${ }^{T M}$ and the open-source plugin IntraLattice [29]. A screenshot of the CAD environment is presented in Figure 2.

The basic ingredients for the design of the lattice wireframe are the definition of the design space, the type of unit cell to be used, grid resolution of the lattice, and strut morphing options, see Figure 1a. Besides using a simple box-shape, IntraLattice allows the user to create conformal lattices that adopt the shape of complex design domains, such as the interior of a boundary-represented (B-rep) volume, the volume spanned by two bounding freeform surfaces, or a cylinder. The design domain is then populated with unit cells to create the lattice topology. A wide range of pre-defined unit cell layouts is provided by IntraLattice, but also user-defined unit cells can be used by drawing the unit cell topology as an assembly of struts in a cube. The wireframe topology can be customized by defining the grid resolution in terms of the number of cells to be used in $u$-, $v$-, $w$-parametric directions or the size of the unit cell. In this way, the nodes of the lattice are transformed such that they conform with the design domain, but by default the struts that connect two nodes remain straight. The struts themselves can also be morphed such that they conform with the freeform boundary and parametric iso-lines of the design domain, which are for instance given through the parametric definition of the surfaces that span the design domain. In this wireframe representation of the lattice, each strut is represented as 3-dimensional NURBS curve, which can be exported in any CAD format and a specific XML format, which we use to interface with our simulation software (see below).

For the solidification of the wireframe, see Figure $1 b$, it is then necessary to define the circular cross-sections of the struts. IntraLattice allows the user to define either a homogeneous, constant strut radius for the whole lattice, or heterogenous, graded radii, which, for instance, can be varied linearly along the $x$-, $y$-, $z$-directions in a Cartesian coordinate system or along the $r$-, $\theta-, z$-axis in a cylindrical coordinate system. It is also possible to use user-defined heterogenous radii, which, for instance, could assign a grading within each unit cell. It should be noted that the grading of radii is not defined per strut, which could yield discontinuous radii at nodes, but in

terms of a continuously varying field, which means that the radii at a node are all equivalent and the radius along a strut is also variable. Likewise, it is also possible to define a heterogenous material distribution for the struts, 
as demonstrated in our previous work [22], where multi-material 3D printing was used to realize spatiallyvariable material distributions in terms of Young's modulus.

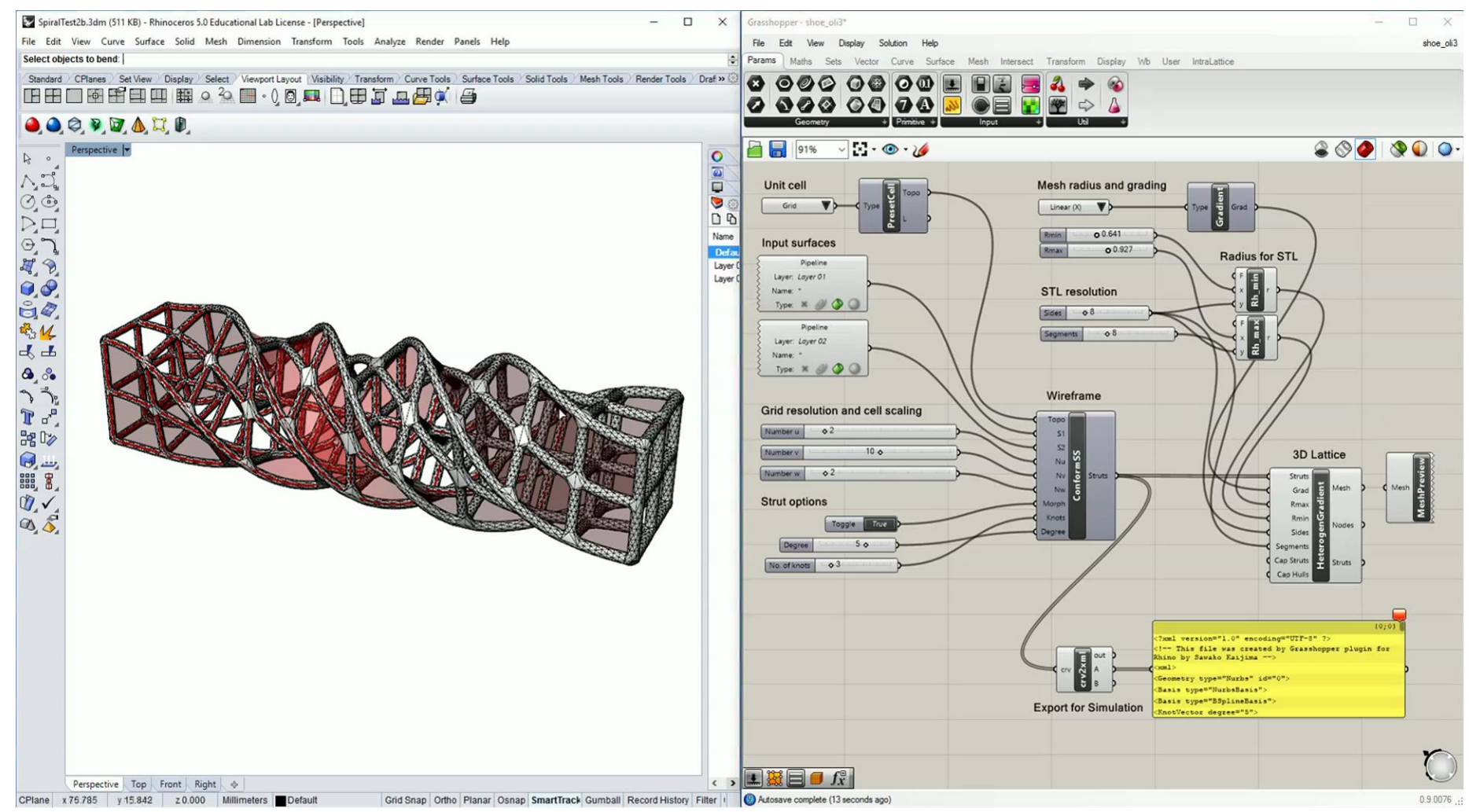

Figure 2. Computer-aided design tool for soft lattice structures. The left window shows the main Rhino ${ }^{\circledR}$ application with the final STL representation of a lattice, which is conformal to the two twisted surfaces shown in transparent red color. The right window shows the Grasshopper ${ }^{T M}$ algorithmic design environment that is used to generate the lattice based on several input parameters.

\subsubsection{STL file generation for manufacture lattice structures}

In principle, the definition of strut radii already yields a solid representation of the lattice. However, for manufacture it is usually necessary to represent the lattice in a mesh format, typically as STL file. Therefore, the implicit description of strut volumes in terms of centerline NURBS curves and cross-sections has to be discretized into a surface triangulation and the node geometries have to be resolved, since struts overlap at joints, see the triangulated lattice representation in Figure 2.

The default approach taken by IntraLattice to discretize the circular strut cross-sections is interpolating them by an inscribed polygon, here a regular hexagon. However, as can be seen in Figure 3, this leads to a significant decrease of the area $A_{n}=r^{2} \frac{n}{2} \sin \left(\frac{2 \pi}{n}\right)$ of the $n$-sided polygon compared to the area $A=\pi r^{2}$ of the original 
circle of radius $r$, and an even higher decrease of the $2^{\text {nd }}$ moment of area, where $I_{x, n}=\frac{n r^{4}}{48}\left(4 \sin \frac{2 \pi}{n}+\sin \frac{4 \pi}{n}\right)$ and $I_{x}=\frac{\pi r^{4}}{4}$. The relative error resulting from this interpolation is $13 \%$ for the area and $24 \%$ for the $2^{\text {nd }}$ moment of area for the regular hexagon, which has significant impact on the mechanical behavior of the lattice structure, as is shown later in the Results section, since the tensile stiffness of a strut is proportional to $A$ and the bending stiffness proportional to $I_{x}$. With increasing number of polygon sides $n$ these errors decrease, but this leads to a higher number of triangles required in the STL file and increased file size, which is not desirable for large, complex lattice structures. Alternatively, we propose to discretize the circles of radius $r$ and area $A$ as polygons of equivalent area, i.e., regular $n$-sided polygons with an increased circumferential radius $\hat{r}=r \sqrt{\frac{2 \pi / n}{\sin (2 \pi / n)}}$. As shown in Fig. 3, this also significantly reduces the error in the $2^{\text {nd }}$ moment of area and results in $3 \mathrm{D}$ printed lattices with the desired mechanical behavior, see Results section.

To implement our design to manufacture framework in the Grasshopper ${ }^{T M}$ design environment, we have introduced into IntraLattice the capabilities to specify the number of polygonal sides and modify the circumferential radius for solidification, see "Radius for STL" and "STL resolution" components in the Grasshopper $^{T M}$ window in Figure 2. Furthermore, we have added an interface of the CAD environment to our nonlinear simulation program described below in Section 2.2 through the NURBS representation of the lattice wireframe, as well as the extraction of 3D node parameters discussed below in Section 2.2.3.

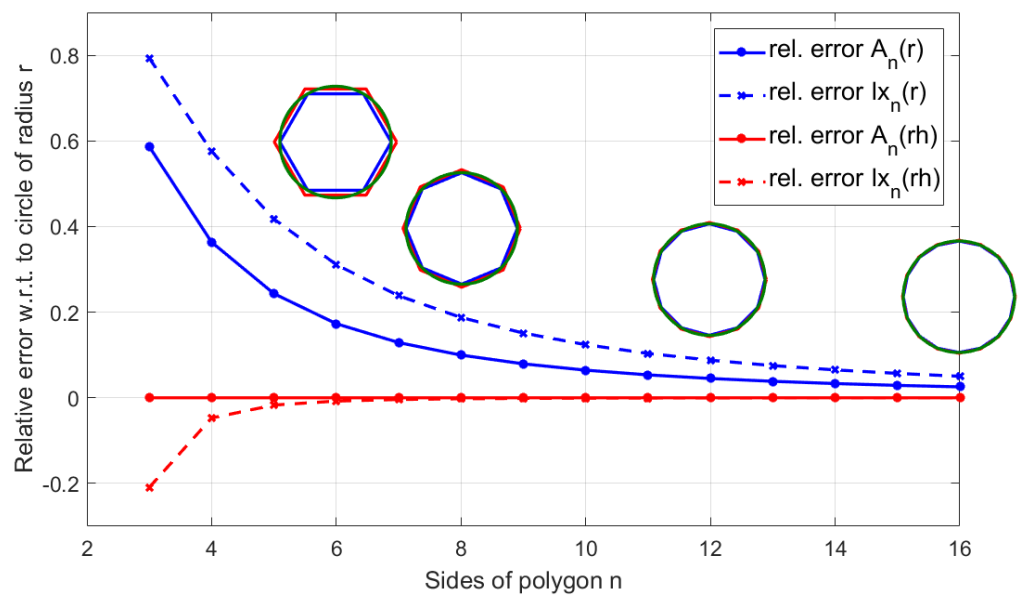

Figure 3. Relative errors in area $\left|A-A_{n}\right| / A$ and $2^{\text {nd }}$ moment of area $\left|I_{x}-I_{x, n}\right| / I_{x}$ due to discretization of circular cross-section of radius $r$ by inscribed $n$-sided polygon (blue lines) and $n$-sided polygon with adjusted circumferential radius $r_{h}$ and equivalent area (red lines). Circular cross-section (green), inscribed polygons (blue) and polygons with same area (red) are shown for $n=6,8,12,16$. 


\subsection{Mechanical modelling and nonlinear simulation of soft lattice structures}

Being able to design lattice structures with conformal freeform shape and graded strut properties, we want to model and simulate their mechanical deformation behavior and enable a simulation-based design approach for such structures before their actual physical realization through 3D printing. Since our focus is on soft lattice structures, we assume that they deform elastically even for large deformations and strains, i.e., no plastic deformation and fracture occur. Since modelling the lattices as nonlinear elastic solids and discretizing them with a nonlinear finite element method is computationally too expensive, we model the lattices as assemblies of nonlinear 3D rods and discretize them using an isogeometric collocation method [27], which allows a direct integration of simulation, which is implemented into an in-house $\mathrm{C}++$ program, with the $\mathrm{CAD}$ approach and balances accuracy with computational efficiency.

\subsubsection{Mechanical modeling}

For the mechanical modelling of a strut, we use the Cosserat rod theory, which is a nonlinear, geometrically exact, elastic 3D beam model that accounts for tension and shear deformation and is thus suitable for modelling both thick and thin 3D beams [30]. The Cosserat rod model is based on representation of the rod as a framed curve, i.e. a rod is described by its centerline, which is a spatial curve $\boldsymbol{r}(s):[0, L] \rightarrow \mathbb{R}^{3}$ and a frame $\boldsymbol{R}(s):[0, L] \rightarrow S O(3)$, which describes the orientation of the cross-sections along the centerline and can be associated with a rotation matrix $\boldsymbol{R}(s)=\left(\boldsymbol{d}_{1}(s), \boldsymbol{d}_{2}(s), \boldsymbol{d}_{3}(s)\right) \in \mathbb{R}^{3 \times 3}: \boldsymbol{R}^{\top} \boldsymbol{R}=\boldsymbol{I}$, see Figure 4a. This completely determines the kinematic configuration of the rod, which is governed by the equilibrium equations of linear and angular momentum (here, ${ }^{\prime}=d / d s$ is the arc length derivative):

$$
\begin{gathered}
\boldsymbol{n}^{\prime}+\widehat{\boldsymbol{n}}=\mathbf{0}, \\
\boldsymbol{m}^{\prime}+\boldsymbol{r}^{\prime} \times \boldsymbol{n}+\widehat{\boldsymbol{m}}=\mathbf{0}, \quad \forall s \in(0, L),
\end{gathered}
$$

as well as boundary conditions for end point positions, orientations, forces and moments. Here, $\boldsymbol{n}=\boldsymbol{R} \boldsymbol{\sigma}$ and $\boldsymbol{m}=\boldsymbol{R} \boldsymbol{\chi}$ represent the internal forces and moments of the rod. External forces and moments are given by $\widehat{\boldsymbol{n}}$ and $\widehat{\boldsymbol{m}}$. The stresses $\boldsymbol{\sigma}=\boldsymbol{C} \boldsymbol{\varepsilon}$ and $\boldsymbol{\chi}=\boldsymbol{D} \boldsymbol{\kappa}$ are determined through linear elastic constitutive laws using the geometrically nonlinear translational strains $\varepsilon=R^{\top} \boldsymbol{r}^{\prime}-\boldsymbol{e}_{3}$ and rotational strains $\boldsymbol{\kappa}=\left[\boldsymbol{R}^{\prime \top} \boldsymbol{R}\right]_{\times}$. The geometric and material properties of the rod cross-sections, i.e. area $A, 2^{\text {nd }}$ moment of area $I$, Young's modulus $E$, and Poisson's ratio $v$, enter the formulation through the constitutive matrices $\boldsymbol{C}$ and $\boldsymbol{D}$. The elastic energy of the rod is given by $U=\frac{1}{2} \int_{0}^{L} \boldsymbol{\varepsilon}^{T} \boldsymbol{\sigma}+\boldsymbol{\kappa}^{T} \chi d s$, which can for instance be used to calculate energy absorption. 


\subsubsection{Numerical simulation}

For the numerical discretization and computational simulation of the governing equations of the Cosserat rod model we use an isogeometric collocation method [27]. It provides an accurate and efficient numerical discretization of the model and enables a seamless integration of the simulation method with the design approach through the concept of isogeometric analysis [31], i.e. a consistent representation of geometry as B-Spline and NURBS curves $\boldsymbol{r}(s)=\sum_{i=1}^{n} N_{i}(s) \boldsymbol{r}_{i}$, which are directly imported from the wireframe lattice design. Here, $N_{i}$ are B-Spline or NURBS basis functions and $\boldsymbol{r}_{i}$ the control points of the curve, see Figure $4 \mathrm{~b}$. The rotation matrices are parameterized as $\boldsymbol{R}(s) \equiv \boldsymbol{R}(\boldsymbol{q}(s))$ with unit quaternions $\boldsymbol{q}(s)=\sum_{i=1}^{n} N_{i}(s) \boldsymbol{q}_{i},\|\boldsymbol{q}(s)\|=1$. To determine the unknown control points of the centerline and quaternion curves $\overrightarrow{\boldsymbol{x}}=\left(\boldsymbol{r}_{i}, \boldsymbol{q}_{i}\right)_{i=1, \ldots, n}$, we apply the concept of isogeometric collocation and evaluate the balance equations of linear and angular momentum, as well as the quaternion normalization condition, at collocation points $\tau_{j}$. For the inner points $\tau_{2}, \ldots, \tau_{n-1}$ this means:

$$
\begin{aligned}
\boldsymbol{f}_{n}\left(\tau_{j}\right) & :=\boldsymbol{n}^{\prime}\left(\tau_{j}\right)+\widehat{\boldsymbol{n}}\left(\tau_{j}\right)=\mathbf{0}, \\
\boldsymbol{f}_{m}\left(\tau_{j}\right) & :=\boldsymbol{m}^{\prime}\left(\tau_{j}\right)+\boldsymbol{r}^{\prime}\left(\tau_{j}\right) \times \boldsymbol{n}\left(\tau_{j}\right)+\widehat{\boldsymbol{m}}\left(\tau_{j}\right)=\mathbf{0}, \\
\boldsymbol{f}_{q}\left(\tau_{j}\right) & :=\boldsymbol{q}\left(\tau_{j}\right)^{\top} \boldsymbol{q}\left(\tau_{j}\right)=0 .
\end{aligned}
$$

Together with the appropriate boundary conditions for forces and moments or displacements and rotations at $\tau_{1}$ and $\tau_{n}$, this yields a nonlinear system of $7 n$ equations for the $7 n$ unknowns, $\overrightarrow{\boldsymbol{f}}(\overrightarrow{\boldsymbol{x}})=\mathbf{0}$. This nonlinear system is solved using Newton's method and using a load-, displacement- or arc-length-controlled incremental nonlinear simulation approach, which allows to capture instabilities and post-buckling effects. Further details on the numerical approach can be found in our previous work [27] and the extension of the method on rod-torod and rod-to-rigid body contact problems in [32].

(a)

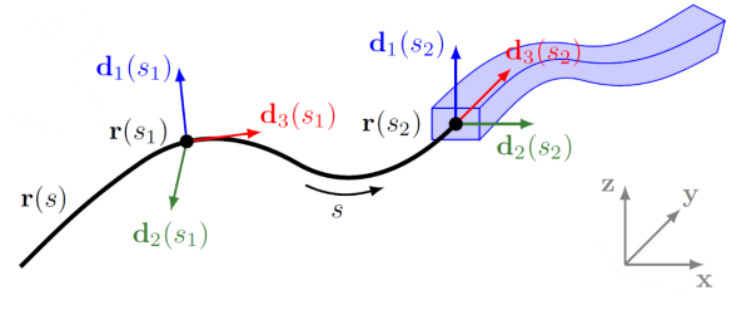

(b)

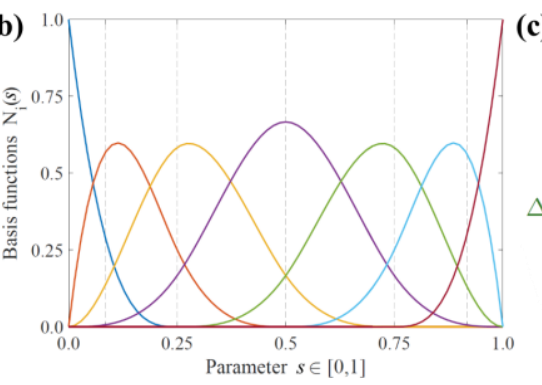

(c)

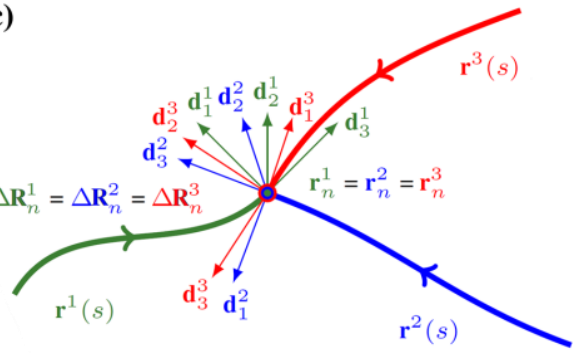

Figure 4. Cosserat rod modelling and isogeometric discretization. (a) Representation of a Cosserat rod by its centerline $\boldsymbol{r}(s)$ and orthonormal frames $\boldsymbol{R}(s)=\left(\boldsymbol{d}_{1}(s), \boldsymbol{d}_{2}(s) ; \boldsymbol{d}_{3}(s)\right)$, defining the cross-section orientations. (b) Example of cubic, $C^{2}$-continuous B-Spline basis functions. (c) Illustration of rigid rod coupling approach 


\subsubsection{Representation of graded radii and joint stiffening approach}

The rod formulation and isogeometric method also allow to accurately represent graded design parameters of the cross-sections, such as a spatially variable material distribution, e.g. in terms of Young's modulus [22], or radius. Here, we focus on lattices with graded cross-section radii, which means that the radii vary not only globally from strut to strut, but also locally within each strut. For instance, a globally linearly varying radius in the $x$-direction would result also in a linearly varying radius of a straight strut in terms of its local parameters $s$, but for a curved strut the dependence on $s$ could be nonlinear. Thus, we parameterize the radius along each strut also as a NURBS curve, i.e. $r:[0, L] \rightarrow \mathbb{R}, r \equiv r(s)=\sum_{i=1}^{n_{r}} N_{i}^{r}(s) r_{i}$. Depending on the scenario, the NURBS basis functions $N_{i}^{r}$ can be chosen differently from those used for the discretization of the rod centerlines.

For the modelling of lattice structures, each strut is represented as a rod and discretized individually. To incorporate connectivity between struts at joints, constraints need to be enforced as boundary conditions on the rods. The most common and straight-forward approach is to model the joints as rigid and enforce that both displacements and angles between rods have to be preserved through the deformation process, i.e., the centerline positions $\boldsymbol{r}$ and change of rotation $\Delta \boldsymbol{R}$ have to be equal for all rods interfacing at the joint, see Figure $4 \mathrm{c}$ and [27]. However, it has been observed that using beam models with the rigid joint modelling approach underestimates the actual stiffness of lattice structures, since lattices have struts with relatively high thicknessto-length ratios and large overlaps at the joints that result in 3D nodes with non-negligible, finite volumes. Thus, so called joint stiffening approaches and semi-rigid joint formulations have been introduced to stiffen the mechanical behavior of struts at joints $[23,28]$. Here, we propose a formulation that stiffens the rods near joints, i.e. thickens them by increasing the radius near the ends of the strut. While similar methods typically require calibration of the stiffening parameters based on experimental results, our approach is solely based on parameters deduced from the actual 3D node geometries, see Figure 5. If required, our parameters could also be fitted to experimental results instead.

We represent the radius $r(s)$ as a quadratic B-Spline curve with $n_{r}=7$, see Figure 5a. The knot vector and control points are calculated from the nominal radii at end points $r^{0}, r^{1}$, the strut length $L$, as well as the normalized radii $\bar{r}^{0}, \bar{r}^{1}$ and offset distances $\bar{\Delta}^{0}, \bar{\Delta}^{1}$ of the $3 \mathrm{D}$ node geometries, see Figure $5 \mathrm{~b}$. Defining the adjusted radii as $r_{0}^{k}=r^{k}\left(1+\left(\frac{r^{k}}{L}\right)^{2}\right)$, the nodal radii as $r_{f}^{k}=r_{0}^{k} \bar{r}^{k}$ and node offset as $r_{d}^{k}=1.4 \bar{\Delta}^{k} r_{0}^{k}$ for each end of the strut, $k=0,1$, we define the knot vector of the radius curve $r(s)$ as $\Xi^{r}=\left\{0,0,0, r_{f}^{0}, r_{d}^{0}, L-\right.$ $\left.r_{d}^{1}, L-r_{f}^{1}, L, L, L\right\}$ and the control points as $\left\{r_{i}\right\}=\left\{r_{f}^{0}, r_{f}^{0}, r_{*}^{0}, \frac{1}{2}\left(r_{f}^{0}+r_{f}^{1}\right), r_{*}^{1}, r_{f}^{1}, r_{f}^{1}\right\}$, where $r_{*}^{0}, r_{*}^{1}$ are determined such that $r^{\prime}\left(\frac{L}{2}\right)=r_{f}^{1}-r_{f}^{0}$. For unmorphed lattices, the normalized radius $\bar{r}=\left(\frac{3}{4 \pi} \bar{V}\right)^{1 / 3}$, where 
$\bar{V}=V / r^{3}$ is the normalized volume of the 3D node, and the normalized offset $\bar{\Delta}=\Delta / r$ can be pre-computed for each type of lattice and joint, see Figure 5c.
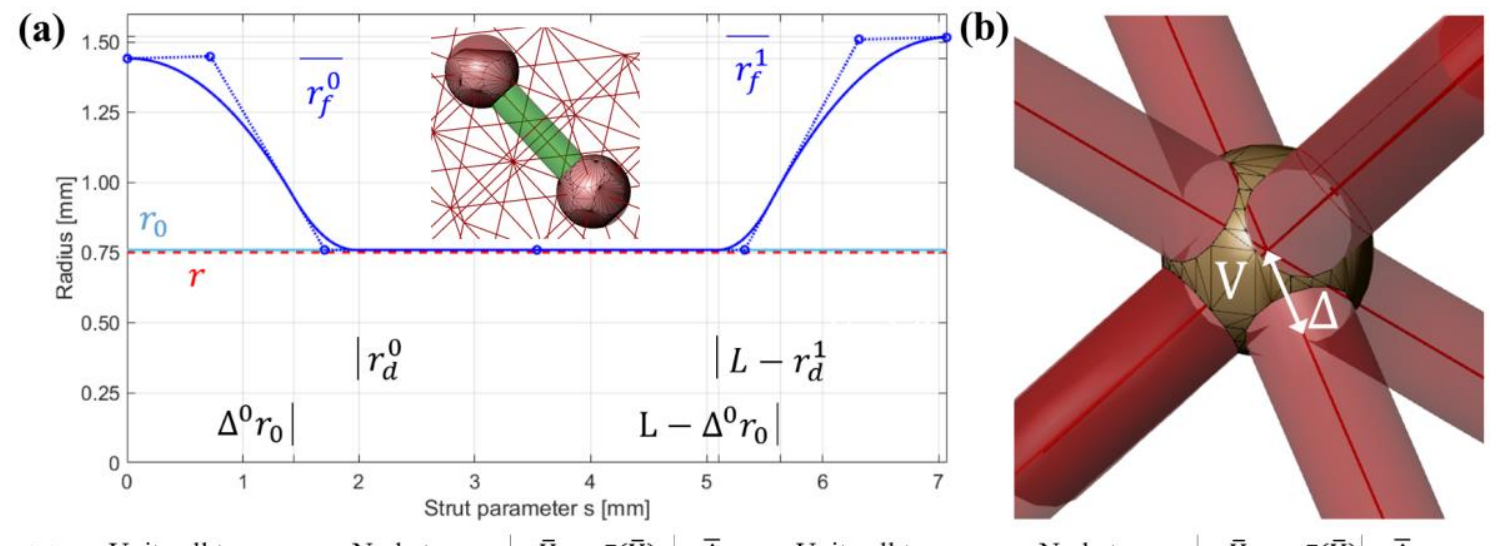

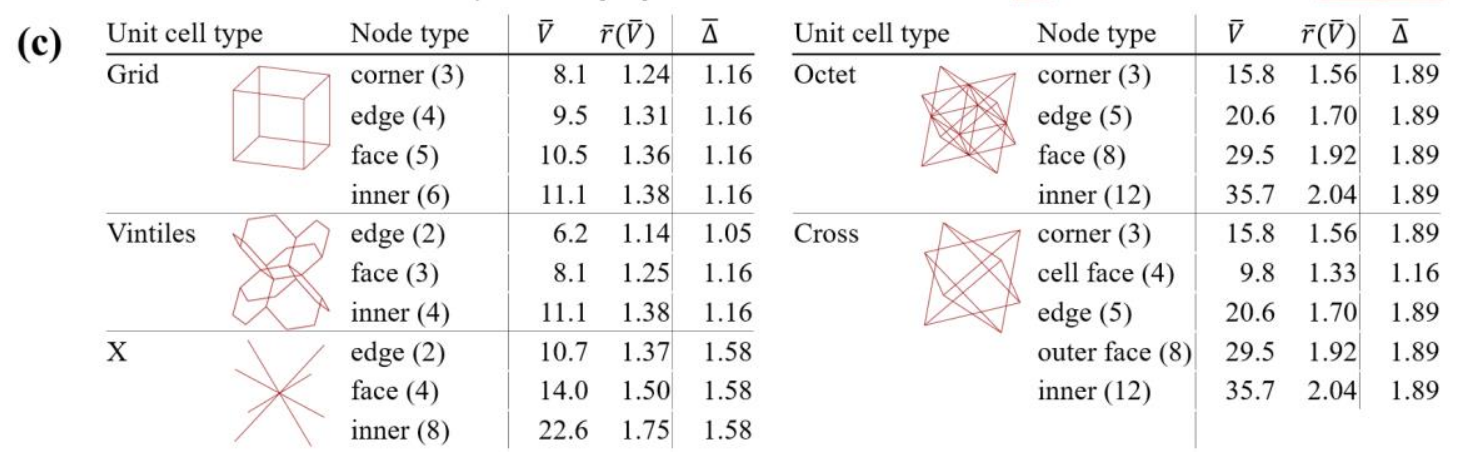

Figure 5. Stiffening of joints through thickening of strut radii. (a) Example of a thickened strut radius curve $r(s)$, here for a strut between nodes of valance 8 and 12 in an Octet lattice with unit cell size $10 \mathrm{~mm}, L=7.072 \mathrm{~mm}$, and nominal radius $r^{0}=r^{1}=0.75 \mathrm{~mm}$. (b) Illustration of 3D node geometry with node volume $V$ and offset $\Delta$. (c) Tabular overview of normalized node volumes $\bar{V}$, radii $\bar{r}$ and offsets $\bar{\Delta}$ for various unit cell and node types (nodal valence in brackets).

\subsubsection{Demonstration of simulation-driven design process}

With the integrated digital design and simulation framework, see Figure 1, that we have presented in detail in Sections 2.1 and 2.2, we facilitate an efficient simulation-driven design process for soft lattice structures. This is now demonstrated with the design of a graded soft lattice structure with specific nonlinear force-displacement behavior, see Figure 6.

In this example, we want to design a lattice structure with a fixed wireframe representation using the "cross" unit-cell type and graded strut diameters. The design goal is that the structure should exhibit (almost) linear force-displacement behavior up to $5 \%$ strain in tension, and then show significant softening for higher applied 
strains. We begin the design process in our design environment in Rhino by specifying the lattice topology as $5 \times 2 \times 2$ cross unit cells of cell size 10x10x10 mm. For the initial design, see Figure $6 \mathrm{a}$, a constant strut radius $r=0.75 \mathrm{~mm}$ is specified for the whole lattice and then a displacement-controlled nonlinear simulation is carried out for tension from 0 to $20 \%$ applied strain. The strain-force curve is plotted in Figure $6 \mathrm{e}$ and shows that the structural response is initially almost linear, but with slight stiffening up to $\sim 8 \%$ strain. Then, the horizontal struts start to buckle and significant softening can be observed. To change the deformation behavior towards our design goal, we now introduce grading of strut diameters along the tensile direction into the design. Thinner struts should buckle at smaller strains and thus move the softening behavior towards the desired applied strain of $5 \%$. However, thinner struts will also lead to a softer overall structural response and to obtain a similar overall stiffness, areas with thicker struts are required, too. To start the simulation driven re-design process, we generate two new designs with graded strut diameters by simply modifying the grading settings in the design environment, see "Mesh radius and grading" in Figure 6f. The first design has a linear grading from $r=0.8$ to $r=0.7 \mathrm{~mm}$, see Figure $6 \mathrm{~b}$, and the second a linear, centered grading from $r=0.8$ to $r=0.7$ to $r=0.8 \mathrm{~mm}$, see Figure 6c. Then, we perform nonlinear simulations for both graded designs, see strain-force curves in Figure 6e. Both structures exhibit the same stiffness in the small strain regime, less stiffening before buckling/softening and soften at smaller strains (at $\sim 7 \%$ and $\sim 6.5 \%$ ) than the initial design. Since the centered gradient behaves slightly better than the linear gradient, we continue by exploring another design with centered grading from $r=$ 0.9 to $r=0.6$ to $r=0.9 \mathrm{~mm}$, see Figure $6 \mathrm{~d}$. The strain-force curve of this graded lattice design in Figure $6 \mathrm{e}$ now fulfills our design goals: same stiffness as the initial design in the small strain regime, linear behavior up to $5 \%$ applied strain and then significant softening. All design parameters for this structure are shown in the snapshot of the design environment in Rhino and Grasshopper in Figure $6 \mathrm{f}$.

This shows that our simulation-driven design process can be efficiently used for the design of soft lattices with desired nonlinear deformation behavior. Within our framework, design alternatives can be easily generated in seconds by changing grading parameters and their performance can be evaluated through the nonlinear beam analysis that only requires seconds to minutes to complete. 
(a)

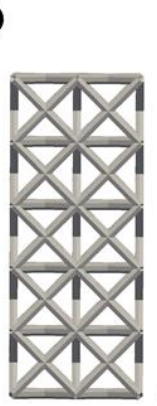

(e)

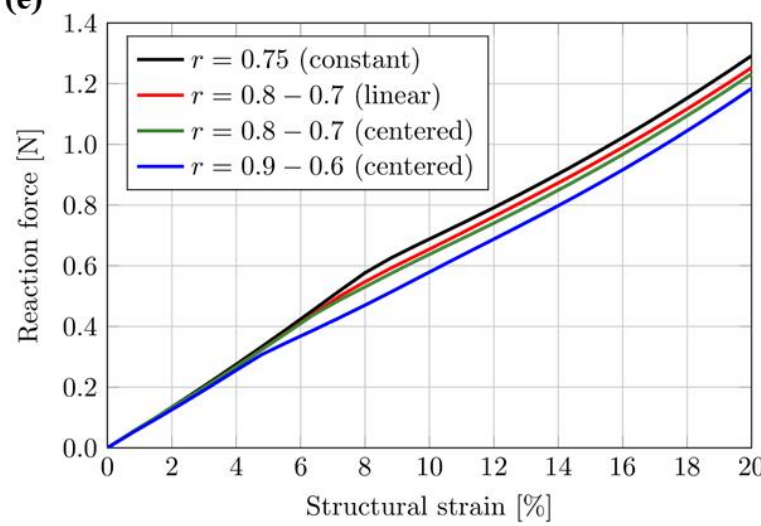

(c)

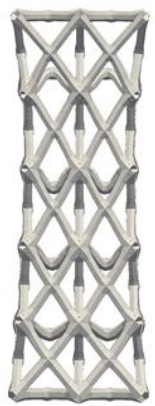

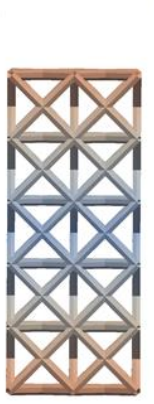

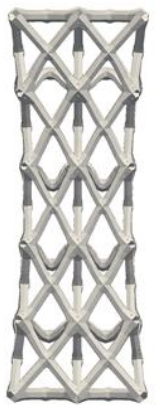

(d)

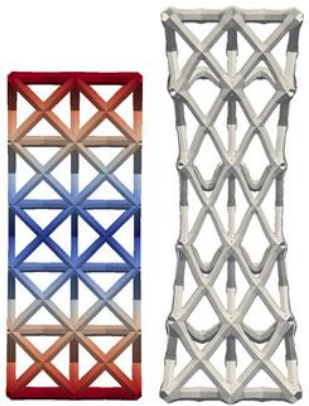

(f)

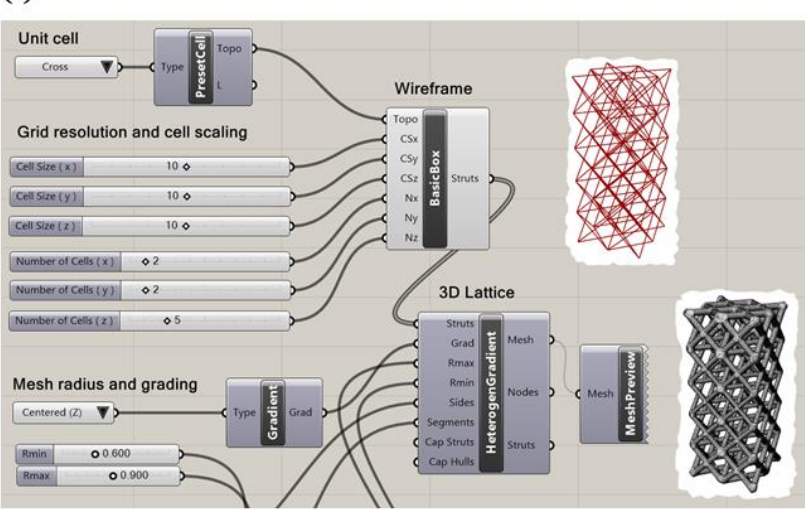

Figure 6. Simulation-driven design of a graded soft lattice structure with specific tensile force-displacement behavior. Four designs of a lattice with $5 \times 2 \times 2$ "cross" cells are investigated, with (a) constant strut radius $r=0.75$, (b) linear grading from $r=0.8$ to $r=0.7$, (c) centered grading from $r=0.8$ to $r=0.7$ to $r=0.8$, and $(d)$ centered grading from $r=0.9$ to $r=0.6$ to $r=0.9$ (left images show undeformed configurations colored by strut radius, right images show deformed configuration at 20\% strain). (e) Strain-force curves of the four lattices in tensile simulation. (f) Snapshot of the design environment in Rhino and Grasshopper for generating lattice configuration (d).

\subsection{Manufacture of soft lattices by PolyJet 3D printing}

We manufacture the soft lattice structures with the commercial material jetting 3D printer Stratasys J750 PolyJet, which deposits and UV-cures liquid polymer resin with voxel-level control of material to fabricate layer wise 3D geometries. As mentioned above, we generate a triangulated boundary representation of the solidified lattice structure in terms of an STL file at the end of our design and simulation pipeline in Rhino ${ }^{\circledR}$, which can be directly imported into the printer software. Alternatively, a voxel-based format could be created and used to generate graded material properties through voxel-level multi-material printing, see [22].

We use elastomeric materials from the Tango family or digital materials with a low volume percentage of glassy polymer material from the Vero family. In particular, we use both TangoBlackPlus and TangoPlus, for which we measured Young's moduli of $E=0.47 \mathrm{MPa}$ and $E=0.45 \mathrm{MPa}$, respectively, as well as the digital material 
DM2160 from TangoBlackPlus and VeroPureWhite with $E=2.0 \mathrm{MPa}$. The measurement data for the material characterization can be found in the Appendix. For all materials, a Poisson's ratio of $v=0.45$ and mass density $\rho=1,150 \mathrm{~kg} / \mathrm{m}^{3}$ are used in the simulations $[18,33]$. Since the failure strains of these 3D printed materials are beyond $100 \%$ [33], we model them as elastic even for large structural strains of $20 \%$ and more. As mentioned above, within the geometrically nonlinear beam formulation we employ the commonly used linear elastic material model to approximate the hyperelastic material behavior exhibited by soft materials. The quality of this assumption is further discussed within the following results section.

The PolyJet 3D printing process uses sacrificial material to support any kind of overhanging structures and also encapsulates all surfaces in a thin layer of support material, which makes it practically impossible to mechanically remove the support material after printing. However, using the recently introduced soluble support material SUP706, sacrificial materials can be easily dissolved. This process typically takes between $1 / 2-2$ days in a water bath with $2 \% \mathrm{NaOH}$ and $1 \% \mathrm{NaSiO}_{3}$ solution, heated to $45^{\circ} \mathrm{C}$. In our experiments, we did not observe any significant change of integrity of the structures or material properties through the dissolution process. Indeed, this process made the fabrication of 3D soft lattice structures with arbitrarily complex unit cell types and shapes feasible.

Since the resolution of the printer, i.e. the voxel size, is $43 \times 86 \times 16 \mu \mathrm{m}$, features larger than $0.1 \mathrm{~mm}$ can be fabricated and grading of strut radii is accurately realized through the manufacturing process, as the smallest radius used in our examples and applications, see below, is $0.5 \mathrm{~mm}$. 


\section{Results and discussion}

Having introduced the computational design, simulation and manufacture framework for soft lattice structures in detail, we now validate the simulation approach against mechanical testing experiments for several lattice structures with various unit cell types and graded radii. We also introduce more complex examples of conformal freeform lattices, a twisted structure and a shoe sole design, which highlight the usability of our approach for practical applications.

\subsection{Experimental validation of workflow}

For the experimental validation of our design-to-manufacture workflow, we carry out mechanical tension and compression tests with 3D printed lattices, as well as numerical simulations with the above-presented approach.

The results for a column-like lattice structures with uniform strut radii are reported in Figure 7, showing initial designs, strain-force curves, as well as snapshots at $20 \%$ strain from experiment and simulation. Various unit cell types, i.e., "Vintiles" (Figure 7a), "Cross" (Figure 7b), "Octet" (Figure 7c), and "X" (Figure 7d+e) are studied. Figure 7a-c compares the results for compression and tension tests for lattices with $5 \times 2 \times 2$ unit cells of cell size $c=10 \mathrm{~mm}$ and radius $r=0.75 \mathrm{~mm}$, printed with TangoBlackPlus material. In Figure $7 \mathrm{~d}+\mathrm{e}$ we compare the tension behavior for " $X$ " type lattices printed with TangoPlus material, in (d) for $5 \times 2 \times 2$ cells of size $c=10 \mathrm{~mm}$ and in (e) for $7 \times 3 \times 3$ cells of size $c=6.5 \mathrm{~mm}$.

We extended the study to functionally graded lattices with spatially varying strut radii, see Figure 8 . Again, we use $5 \times 2 \times 2$ unit cells of size $c=10 \mathrm{~mm}$ and type "Vintiles" (Fig. 8a) and "Cross" (Fig. 8b). The strut radii are cylindrically varying along the $x$-axis, i.e. the longest direction of the beams. From inside to outside, the radius changes from 0.50 to $0.75 \mathrm{~mm}$ for the "Vintiles" case and vice versa for the "Cross" structure.

To be able to carry out mechanical tests, we integrate clamps at both ends of the short beam-like lattice designs, which are printed from Vero materials. The clamps are positioned $50 \mathrm{~mm}$ apart such that they slightly overlap the lattices by a distance equal to strut radius, $r \mathrm{~mm}$ on each side, resulting in good bonding between the Vero and Tango parts when monolithically 3D printed. Since Vero acts rigidly compared to the soft lattices due to its

high Young's modulus of over $1 \mathrm{GPa}$ [33], the clamps help in mounting the lattices in the mechanical testing machine, but do not distort the measurements. To assess the general measurement errors and deviations, we have 3D printed three identical copies of each design and carried out two tests for each of the prints, i.e. a total of six mechanical tests for every design. The figures show the average and extrema of those measurement results. The compression and tension test were performed on an MTS mechanical testing machine with a load cell of $100 \mathrm{~N}$ over a range of $0 \%$ to $20 \%$ structural or engineering strain. 
The "Vintiles" lattices in Figure 7a and Fig. 8a show initially linear behavior in both compression and tension, gradually becoming nonlinear for larger strains in terms of softening in the former and hardening in the latter case due to geometric effects in the lattice. Significant deviations are only recognizable for compression of the graded lattice in Fig. 8a1, where the simulation overestimates the force when compared to experimental results. However, this is likely related to a global buckling mode that can be observed in experiments (bending towards the left side in the photo in Fig. 8a1), which occurs due to inaccuracies in the experimental setup and cannot be reproduced in the simulations.

The "Cross" lattices in Figure 7b and Figure 8b exhibit interesting instabilities, i.e., micro-buckling of struts in the loading direction during compression and of struts perpendicular to the loading direction during tension. The initially linear behavior in both compression and tension is well captured in simulations, but the strains at which local buckling occurs (kinks in the strain-force curves), are mostly over-estimated in the simulations. Nevertheless, the post-buckling response is well captured in terms of nonlinear softening behavior, for both the uniform lattice in Figure $7 \mathrm{~b}$ and the graded lattice in Fig. 8b.

In Figure 7b, we have also plotted the simulation results without our thickening approach (Sim. (no thick.)), which show a considerably softer behavior. Also shown are measurements for structures that were printed with a discretization of the circular cross-sections with only an inscribed hexagon $(\operatorname{Exp} .(n=6))$ and simulations with a smaller radius that corresponds to the equivalent cross-section area $(\operatorname{Sim} .(r=0.68))$. It can be seen that their behavior is considerably softer, which underlines the necessity to discretize the cross-section in the STL files with either a polygon with a sufficiently large number of edges (typically we have used $n=16$ ) or an increased effective radius.

The structural deformation behavior of the "Octet" unit cell type shown in Figure 7c is similar to the "Vinitiles" cells, i.e., nonlinear softening for compression, here induced by micro-buckling of struts, and hardening for tension. The simulations capture this well in both cases and are in good agreement with the experiments. For the structures with " $\mathrm{X}$ " unit cell type investigated in Figure $7 \mathrm{~d}+\mathrm{e}$, agreement is good when using smaller unit cells as shown in Fig. 7e. The simulation results are, however, too stiff with larger unit cells but same dimensions as shown in Fig. 7d. This might be related to a geometric defect that occurs in all our printed samples, the right column of cells (which was printed on top) being skewed.

Overall, the presented experimental and numerical simulations results are in good agreement. Deviations between measurements and simulations could be due to hyperelasticity of the material behavior. Though axial strains observed for individual rods do not exceed 15\% for $20 \%$ applied structural strain, curvature induced strains can be much higher, especially when buckling occurs, as for the "Cross" lattice type, where the determinant of the deformation gradient ranges from 0.56 to 1.30 in the compression case. Other potential 
reasons include uncertainties in the material parameters, especially slight anisotropy of the material, or in the fabricated geometries, e.g., circular or polygonal cross-section becoming jiggered, or defects such as the one in Fig. 7d. No qualitative differences in the accuracy of results could be observed with either graded or uniform strut radii, which shows that our approach is well-suited for simulation of functionally graded lattices.

(a0)

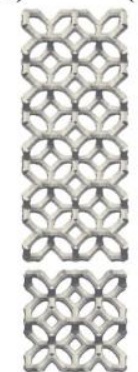

(a1)

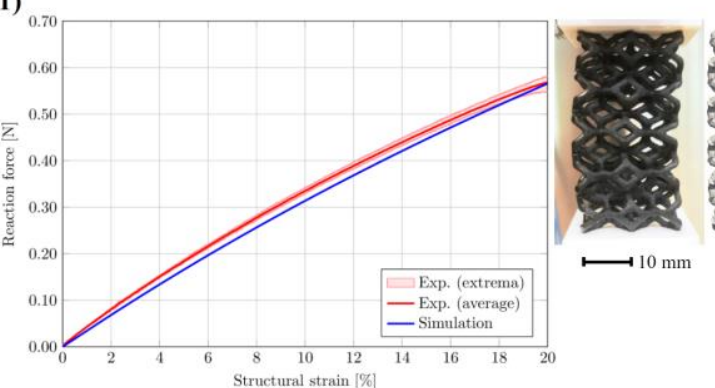

(b0)

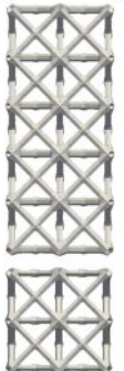

(c0)

(b1)
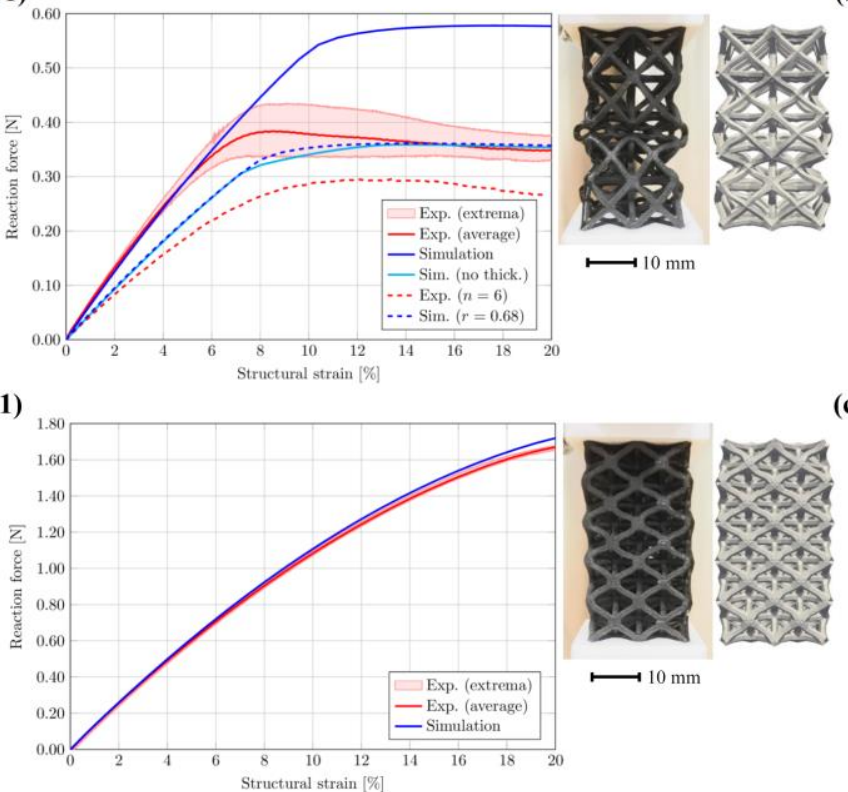

(e0)
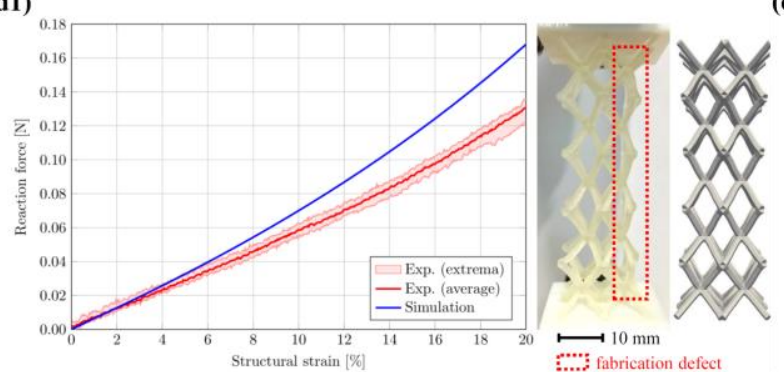

(b2)

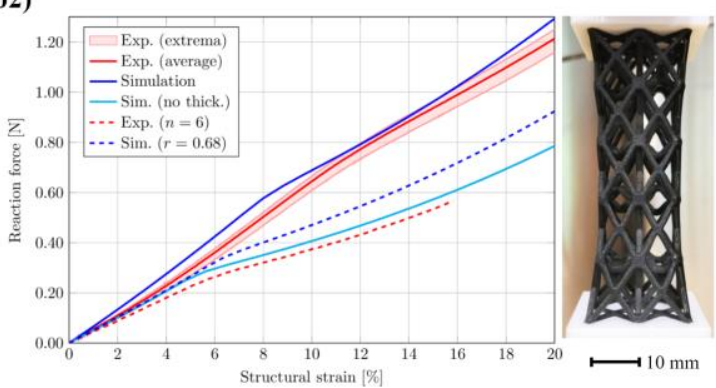

(c2)
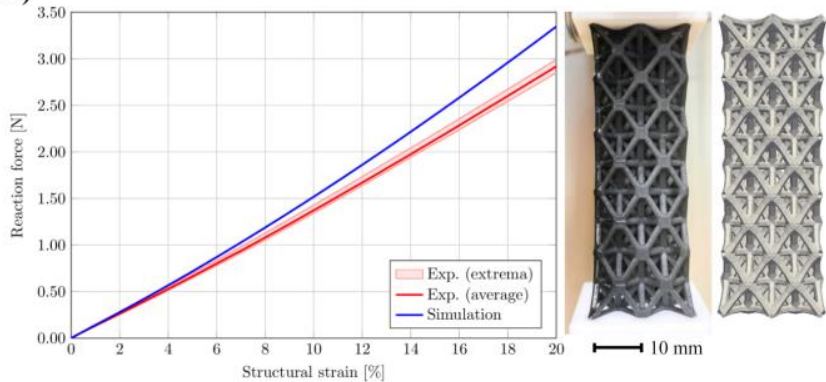

(a2)

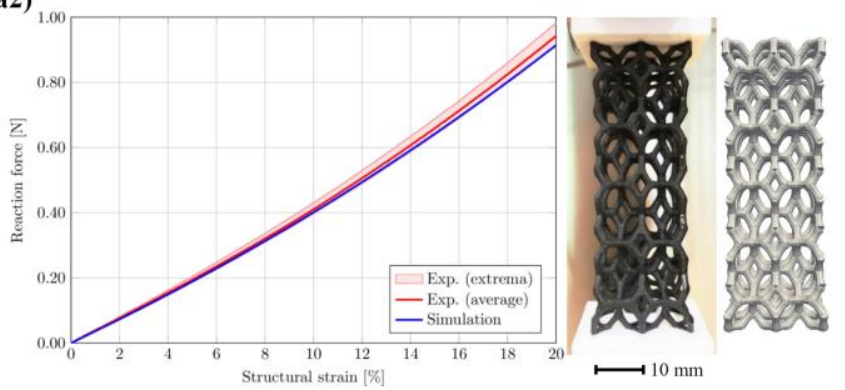

(e1)

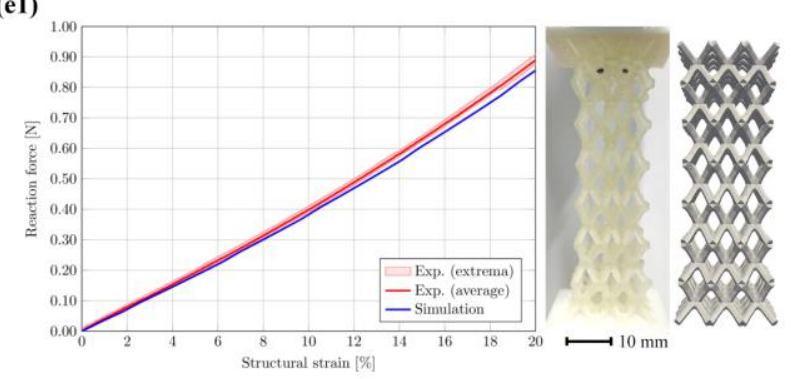

Figure 7. Validation of mechanical testing against simulations for soft lattices with $5 \times 2 \times 2$ cells, cell size $c=10 \mathrm{~mm}$, and uniform strut radius $r=0.75 \mathrm{~mm}$. Column (0) shows $y x$ - and $y z$-views of lattice designs, columns (1) and (2) show strainforce curves and snapshots at $20 \%$ strain for compression and tension, respectively, for lattices with (a) Vintiles, (b) Cross, (c) Octet unit cells. (d) and (e) show only tension for X unit cells with (d) $5 \times 2 \times 2$ cells, $c=10 \mathrm{~mm}, r=0.75 \mathrm{~mm},(e) \mathrm{X}$, $7 \times 3 \times 3$ cells, $c=6.5 \mathrm{~mm}, r=0.75 \mathrm{~mm}$. 
(a0)

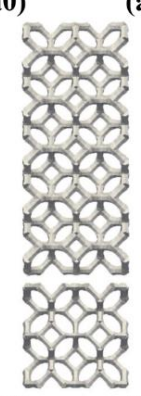

(b0)

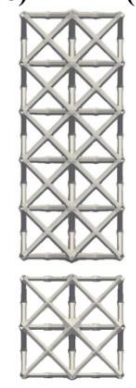

(a1)

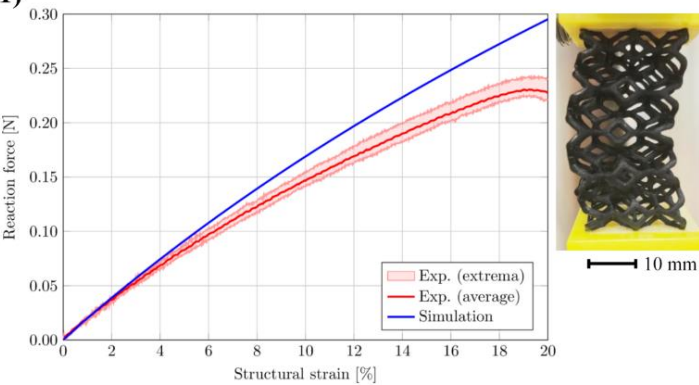

(b1)
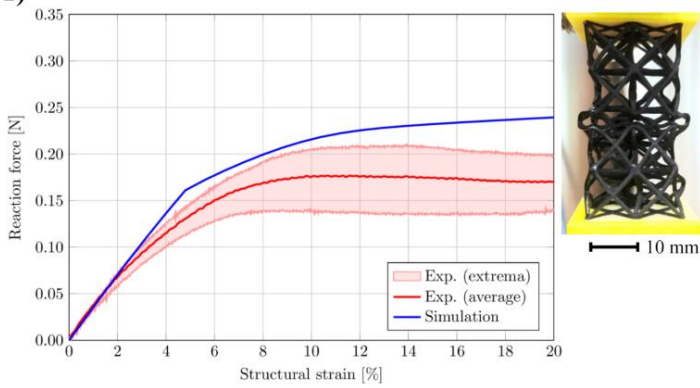

(a2)
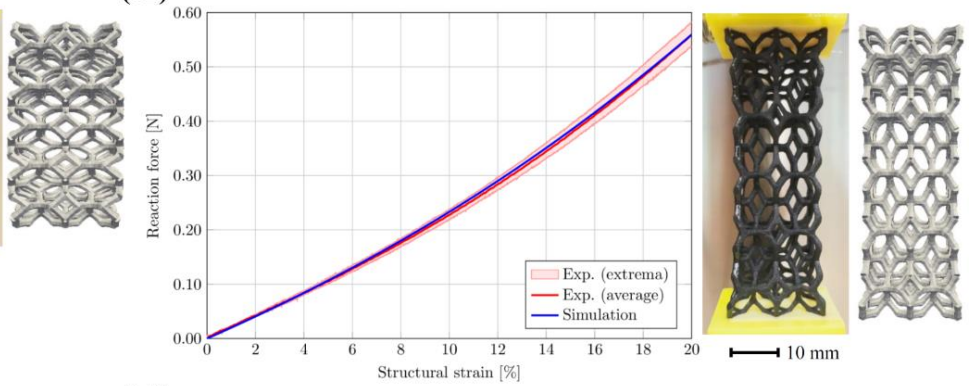

(b2)
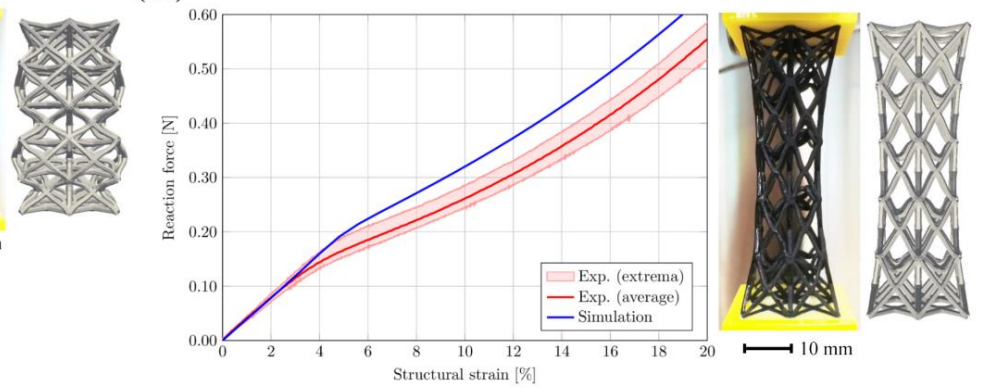

Figure 8. Validation of mechanical testing against simulations for soft lattices with graded strut radii, using $5 \times 2 \times 2$ cells, $c=10 \mathrm{~mm}$. Column (0) shows $y x$ - and $y z$-views of lattice designs, columns (1) and (2) show strain-force curves and snapshots at $20 \%$ strain for compression and tension, respectively, for lattices with $(a)$ Vintiles, $r=0.50-c y l(x)-0.75$ $\mathrm{mm}$ (b) Cross, $r=0.75-\operatorname{cyl}(x)-0.50 \mathrm{~mm}$.

\subsection{Twisted grid structure with curved struts}

Having validated our design and simulation framework, we now investigate an example with curved struts, a twisted lattice with "Grid" unit cells, see Figure 9. Compared to the previous lattices used for validation purposes, the complexity of the design is increased here in several ways: the lattice is defined by a freeform design domain; the struts conform to the domain by becoming curved; and this geometric complexity results in an interesting mechanical behavior, that could not be predicted by standard, linear simulation tools.

The design and manufacture process for the structure is outlined in Figure 9a. First, two rectangular surfaces of 100x20 mm were created, offset $20 \mathrm{~mm}$ from each other. These surfaces are twisted by $2 \pi$ along the $x$-axis, resulting in the intertwined red freeform NURBS surfaces shown in Figure 9a1. Then, a lattice wireframe is generated between those surfaces with 10x2x2 unit cells of type "Grid", i.e. cuboids, and the struts are morphed such that they align with the twisted surfaces, see the green wireframe in Figure 9a1. For the solidification, a constant strut radius of $r=0.75 \mathrm{~mm}$ is specified and an STL file is generated (Figure 9a2). The lattice structure is printed with TangoPlus, along with attached clamps that are printed with VeroPureWhite (Figure 9a3). After dissolving the support material that covered all struts, we obtain the desired soft lattice structure with twisted shape (Figure 9a4). 
We also study the mechanical behavior of the structure and validate the simulation for this complex shape. First, we clamp the structure vertically in the mechanical testing machine, such that it cannot extend when subject to its body weight. The actual shape of the slightly deformed structure is in good agreement with the corresponding simulation, see Figure 9b. Then, we release the lower fixture and the lattice can now freely deform and rotate at its lower end when subject to self-weight plus the weight of the Vero clamp of $0.09 \mathrm{~N}$. In the experiment, shown in Figure 9c, we observe coupling of extension and twist behavior, which results in $12.5 \%$ strain and a rotation of the lower end by $-69^{\circ}$. The numerical results agree well, with a strain of $11 \%$ and a rotation of $-74^{\circ}$. Furthermore, the shapes of deformed struts are also similar in simulation and physical experiment, including features such as buckling of struts and complex bending, which are highlighted using red arrows in Figure 9c.

With the twisted lattice, we have validated our approach also for the design and simulation of geometrically complex, non-intuitive structures that exhibit non-classical mechanical behavior. Here, we particularly benefit from the integration of design with isogeometric simulation, as the exact geometries in terms of NURBS curves are used without any loss of geometric accuracy.

(a1)

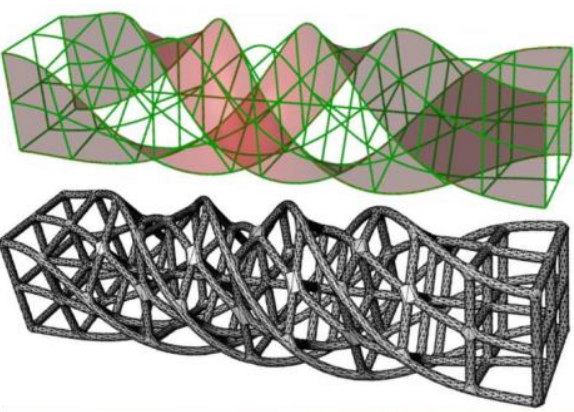

(a3)

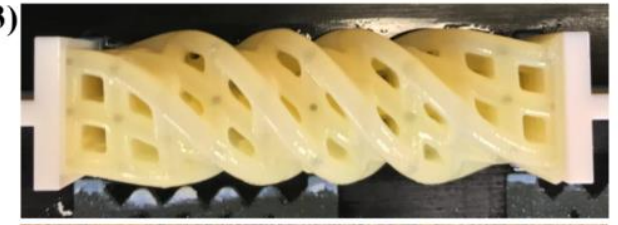

(a4)

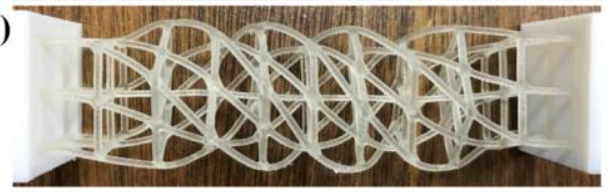

(b)

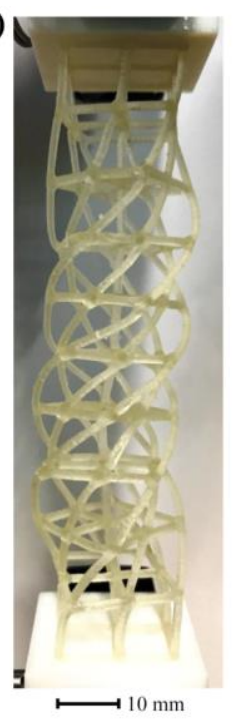

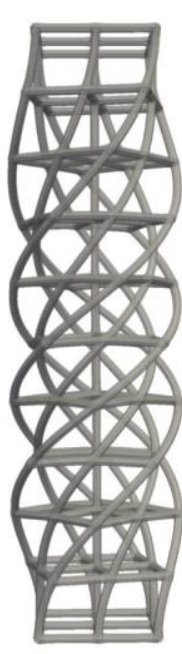

(c)

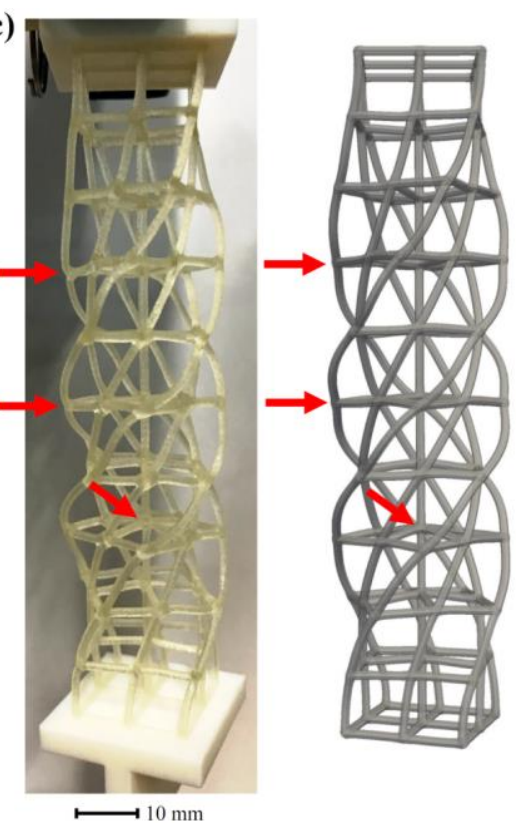

Figure 9. Twisted grid structure. (a) Design of lattice (top to bottom): wireframe (green) with 10x2x2 grid type cells, $c=$ $10 \mathrm{~mm}$ and curved struts, conforming to twisted surfaces (red); solidified lattice with $r=0.75 \mathrm{~mm}$ in STL format; as printed structure with support material; final structure without support material. (b) Lattice is clamped to its original length of $100 \mathrm{~mm}$ and subject to deformation under self-weight (experiment vs. simulation). (c) Lattice is clamped at top and free at bottom, subject to un-twisting deformation under self-weight plus weight of lower clamp (experiment vs. simulation). 


\subsection{Digital design and manufacture of a soft lattice shoe sole}

Finally, we present a digital design to manufacture workflow for a consumer product application with complex geometry and material distribution; a lattice-structured shoe sole motivated by a recent product introduced by Carbon + Adidas, their Futurecraft4D shoe [20]. Such a shoe sole with a tailored soft lattice structured with morphed geometry and graded struts could be easily adapted to different shoe designs and customized not only for aesthetics, but also with an adaptive stiffness distribution, that considers the customer's footprint for individual walking comfort or therapeutic reasons, or performance requirements regarding energy absorption for sports applications.

The detailed workflow with our digital manufacture and design framework is outlined in Figure 10. Again, we start from two freeform surfaces that define the design domain for the lattice generation, the red surfaces in Figure 10a. As mentioned, these surfaces could be easily adapted to represent designs or footprints of a specific person, e.g., acquired from a 3D scan. Then, the wireframe is generated, here using $18 \times 6 \times 2$ cells of type " $\mathrm{X}$ ", which conform to the freeform shape geometries, and solidified with a linear gradient of strut radius from 1.0 to $1.2 \mathrm{~mm}$ from tip to heel (Figure 10b). While this linear grading of the strut radii is chosen arbitrarily, it could reflect the desire for a stiffer response under the heel in a real shoe design. This could also be adapted into a performance goal for a design optimization process. Here, we carry out a simulation of the shoe sole, where the top surface is subjected to increasing, downward-directed displacement, which leads to contact with the rigid ground surface and compression of the lattice (Figure 10c). Then, we introduce an elastic surface on top of the lattice into the design, which simulates the interface with the upper shoe (Figure 10d) and proceed with 3D printing. The printed structure with support material is then shown in Figure 10e and after dissolving in Figure 9f. The lattice was printed with the digital material DM2160 and the top surface with rigid-like VeroPlus material, which is transparent. Figure 10f then illustrates the deformation of the 3D printed lattice shoe sole when a load is applied to the top surface. A higher resolution photo of this lattice shoe sole design, as well as of an alternative design using "Cross" unit cells are shown in Figure 10g.

With this example of a tailored soft lattice shoe sole, we demonstrated the usability of our framework and the potential to incorporate soft lattice structures into specific product applications. Here, we also integrated the soft lattice structure with a stiff, shell-like part, that was realized in a single, monolithic multi-material 3D print, which highlights the benefits of using multi-material material jetting technology for soft lattice-type applications. 

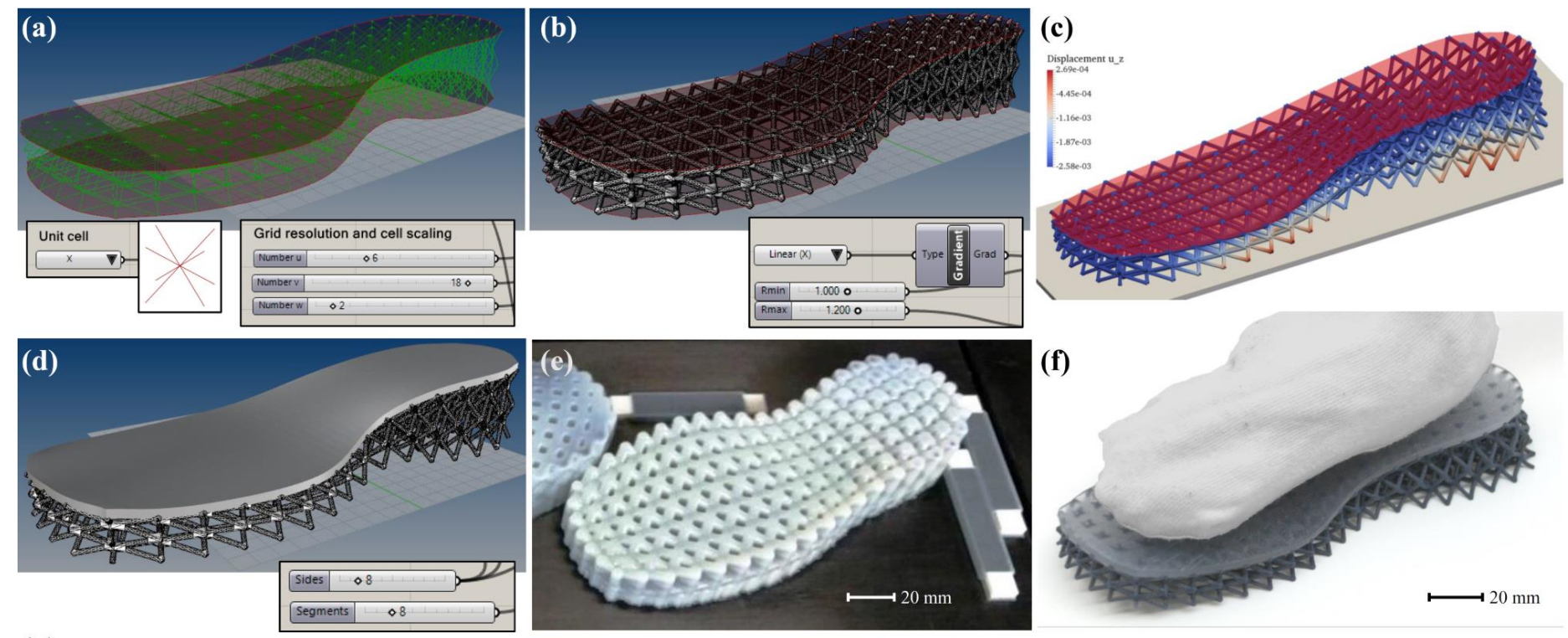

(g)

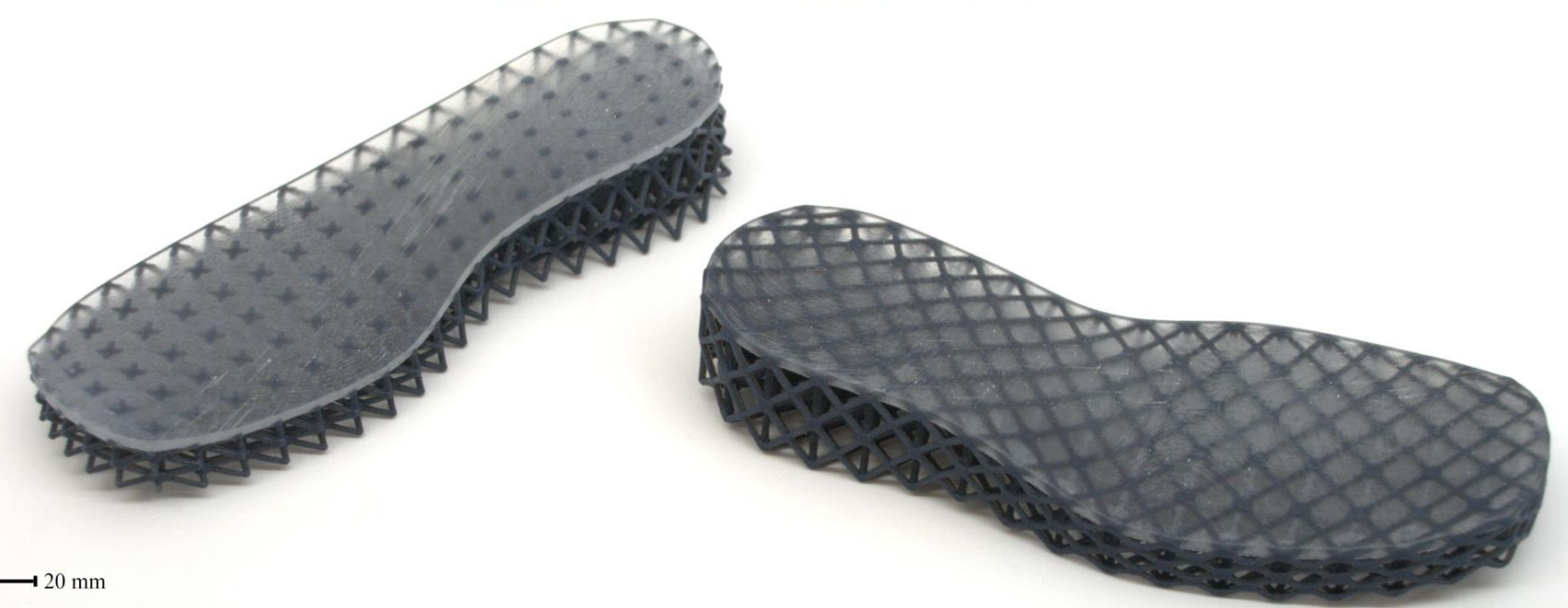

Figure 10. Lattice shoe sole. (a) Lattice wireframe (green) with conformal design given by top and bottom freeform surfaces (red) and 18x6x2 cells of type "X". (b) Solidified lattice with radius linearly increasing from $1 \mathrm{~mm}$ at tip to 1.2 $\mathrm{mm}$ at heel. (c) Simulation with uniform pressure load on top surface and contact on ground surface. (d) STL representation with added top surface plate. (e) As printed lattice with support material (upside down). (f) Deformed soft lattice under load on rigid top surface (transparent). ( $g$ ) Higher resolution photo of the shoe sole with " $\mathrm{X}$ " unit cells (left) and an alternative design with "Cross" type cells (right). 


\section{Conclusion}

We have presented a computational workflow framework for soft lattice structures, which covers the design of soft lattice structures in specific components, nonlinear mechanical simulation of the lattice structures, and fabrication using 3D printing. The $\mathrm{CAD}$ approach allows design of 3-dimensional lattice structures that conform to a freeform design space and have functionally graded properties, here in terms of strut radii. Mechanical simulation is based on nonlinear 3D rod modelling and an efficient isogeometric collocation method, that is based on the exact NURBS geometries of curved struts. The method is enhanced with a joint-stiffening approach, which thickens the strut radii near joints, based on parameters deduced from the actual 3D node geometries. We have validated the design and simulation method by 3D printing soft lattices on a PolyJet multimaterial printer with soluble support material. Furthermore, we have demonstrated that complex nonlinear behavior including instabilities, post-buckling, and coupling of extension and twist modes can be predicted by our simulations, exceeding the capabilities of existing tools, and highlighted the applicability of the framework by applying it to a specific application - a shoe sole with tailored mechanical behavior.

To further enhance the overall framework in the future, we plan to extend it to a computational design optimization approach, which can be used to obtain optimal grading of individual strut radii or even curved strut shapes. Furthermore, functional grading of material properties through voxel-based multi-material printing can be easily integrated into our framework. To further improve the accuracy of the simulation method, hyperelastic material models could be implemented.

\section{Acknowledgements}

The authors acknowledge support from the SUTD Digital Manufacturing and Design (DManD) Centre, supported by the Singapore National Research Foundation. We would like to thank B. Narayanan, Y. J. Teoh and R. Junsly from SUTD for discussions and help with software, simulations and 3D printing. We also acknowledge Professor D. Rosen from SUTD for valuable discussions.

\section{Author Disclosure Statement}

No competing financial interests exist. 


\section{Appendix}

Figure A1 shows the stress-strain curves obtained for the characterization of the materials used in this work with the commercial material jetting 3D printer Stratasys J750 PolyJet, namely TangoBlackPlus, TangoPlus and DM2160, which is a mix of TangoBlackPlus and VeroPureWhite [33]. The curves are obtained by performing uniaxial tension tests on 3D printed samples of 50x10x4 mm size on a MTS Criterion universal testing machine with a $100 \mathrm{~N}$ load cell. Since the two materials from the Tango family exhibit only mildly hyperelastic behavior with softening for higher strain rates, we determine the Young's modulus by averaging the results from 5-6 samples in the strain range from 1\%-3\%. However, for DM2160 hyperelastic material behavior is more prominent and we chose the Young's modulus by averaging from 5\%-15\% strain.

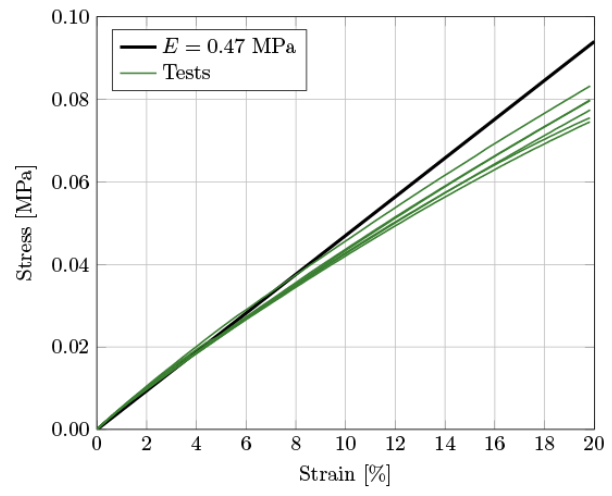

(a) TangoBlackPlus

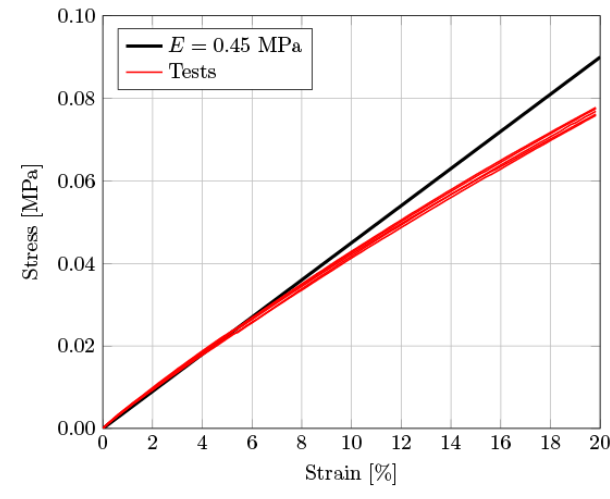

(b) TangoPlus

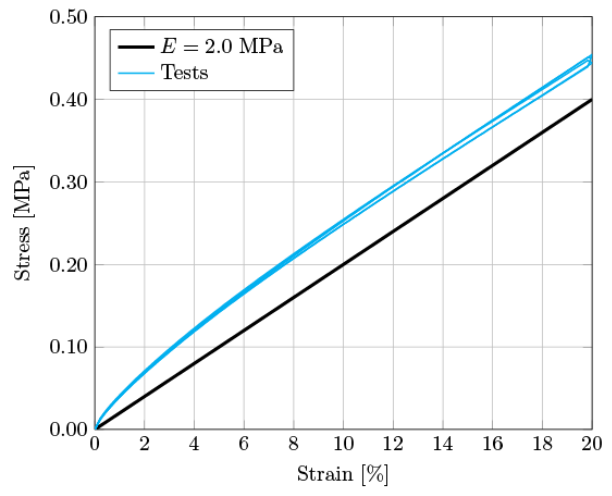

(c) DM2160

Figure A1. Stress-strain curves for uniaxial tension tests of (a) TangoBlackPlus, (b) TangoPlus and (c) DM2160. Linear elastic response is also plotted for Young's modulus values $E=0.47 \mathrm{MPa}, E=0.45 \mathrm{MPa}$, and $E=2.0 \mathrm{MPa}$, respectively. 


\section{References}

[1] L. E. Murr, S. M. Gaytan, D. A. Ramirez and et al., "Metal Fabrication by Additive Manufacturing Using Laser and Electron Beam Melting Technologies," J. Mater. Sci. Technol., vol. 28, no. 1, pp. 1-14, 2012.

[2] J. Wallach and L. Gibson, "Mechanical behavior of a three-dimensional truss material," Int. J. Solids Struct., vol. 38, no. 40-41, pp. 7181-7196, 2001.

[3] R. Gümrük and R. A. W. Mines, "Compressive behaviour of stainless steel micro-lattice structures," Int. J. Mech. Sci., vol. 68, pp. 124-139, 2013.

[4] D. W. Rosen, "Computer-Aided Design for Additive Manufacturing of Cellular Structures," Comput. Aided Des. Appl., vol. 4, no. 5, pp. 585-594, 2007.

[5] S.-I. Park, D. W. Rosen, S.-K. Choi and C. E. Duty, "Effective Mechanical Properties of Lattice Material Fabricated by Material Extrusion Additive Manufacturing," Addit. Manuf., Vols. 1-4, pp. 12-23, 2014.

[6] G. Dong, Y. Tang and Y. Zhao, "A Survey of Modeling of Lattice Structures Fabricated by Additive Manufacturing," ASME J. Mech. Des., 2017.

[7] L. Hao, D. Raymont, C. Yan, A. Hussein and P. Young, "Design and Additive Manufacturing of Cellular Lattice Structures," in Proc. 5th Int. Conf. Adv. Res. Virtual Rapid Prototyping, Leira, Portugal, 2011.

[8] Y. Jiang and Q. Wang, "Highly-stretchable 3D-architected Mechanical Metamaterials," Sci. Rep., vol. 6, p. 34147, 2016.

[9] T. Chen, J. Mueller and K. Shea, "Integrated Design and Simulation of Tunable, Multi-State Structures Fabricated Monolithically with Multi-Material 3D Printing," Sci. Rep., vol. 7, p. 45671, 2017.

[10] J. Liu, T. Gu, S. Shan, S. H. Kang, J. C. Weaver and K. Bertoldi, "Harnessing Buckling to Design Architected Materials that Exhibit Effective Negative Swelling," Adv. Mater., vol. 28, no. 31, pp. 6619-6624, 2016.

[11] Y. Chen, F. Qian, L. Zuo, F. Scarpa and L. Wang, "Broadband and multiband vibration mitigation in lattice metamaterials with sinusoidally-shaped ligaments," Extreme Mechanics Letters, vol. 17, pp. 24-32, 2017.

[12] Y. Chen, T. Li, J. Zian, F. Scarpa, C.-W. Yao and L. Wang, "3D printed hierarchical honeycombs with shape integrity under large compressive deformations," Materials \& Design, vol. 137, pp. 226-234, 2018.

[13] N. G. Cheng, A. Gopinath, L. Wang, K. Iagnemma and A. E. Hosoi, "Thermally Tunable, Self-Healing Composites for Soft Robotic Applications," Macromol. Mater. Eng., vol. 299, no. 11, pp. 1279-1284, 2014. 
[14] Z. Ding, C. Yuan, X. Peng, T. Wang, H. J. Qi and M. L. Dunn, "Direct 4D printing via active composite materials," Sci. Adv., vol. 3, p. e1602890, 2017.

[15] Z. Ding, O. Weeger, H. J. Qi and M. .. Dunn, "4D Rods: 3D Structures via Programmable 1D Composite Rods," Mater. Des., vol. 137, pp. 256-265, 2018.

[16] A. Clausen, G. Wang, J. S. Jensen, O. Sigmund and J. A. Lewis, "Topology Optimized Architectures with Programmable Poisson's Ratio over Large Deformations," Adv. Mater., vol. 27, no. 37, pp. 5523-5527, 2015.

[17] S. Babaee, J. Shim, J. C. Weaver, E. R. Chen, N. Patel and K. Bertoldi, "3D Soft Metamaterials with Negative Poisson's Ratio," Adv. Mater., vol. 25, no. 36, pp. 5044-5049, 2013.

[18] Y. M. Xie, X. Yang, J. Shen and e. al., "Designing orthotropic materials for negative or zero compressibility," Int. J. Solids Struct., vol. 51, pp. 4038-4051, 2014.

[19] D. K. Patel, A. H. L. M. Sakhaei, B. Zhang, Q. Ge and S. Magdassi, "Highly Stretchable and UV Curable Elastomers for Digital Light Processing Based 3D Printing," Adv. Mater., vol. 29, no. 15, p. 1606000, 2017.

[20] Carbon 3D, "The perfect fit: Carbon + adidas collaborate to upend atheletic footware," [Online]. Available: http://www.carbon3d.com/stories/adidas/.

[21] C. Wyman, "Why Stratasys' New SUP706 Soluble Support Material Is Ideal for Intricate Parts," Stratasys Ltd., 1 Dec 2015. [Online]. Available: http://blog.stratasys.com/2015/12/01/sup706-soluble-support-material/.

[22] O. Weeger, Y. Kang, S.-K. Yeung and M. Dunn, "Optimal Design and Manufacture of Active Rod Structures with Spatially Variable Materials," 3D Print. Addit. Manuf., vol. 3, no. 4, pp. 204-215, 2016.

[23] S.-I. Park and D. Rosen, "Homogenization of Mechanical Properties for Additively Manufactured Periodic Lattice Structures Considering Joint Stiffening Effects," in ASME IDETC/CIE 2016, Charlotte, NC, 2016.

[24] D. Cioranescu and J. Saint-Jean-Paulin, "Lattice-Type Structures," in Homogenization of Reticulated Structures, New York, Springer, 1999, pp. 71-142.

[25] L. J. Gibson and M. F. Ashby, Cellular Solids - Structure and Properties, Cambridge University Press , 1997.

[26] K. Suzuki and N. Kikuchi, "A homogenization method for shape and topology optimization," Comput. Methods Appl. Mech. Eng., vol. 93, no. 3, pp. 291-318, 1991.

[27] O. Weeger, S.-K. Yeung and M. Dunn, "Isogeometric collocation methods for Cosserat rods and rod structures," Comput. Methods. Appl. Mech. Eng., vol. 316, pp. 100-122, 2017.

[28] M. Sekulovic and R. Salatic, "Nonlinear analysis of frames with flexible connections," Comput. Struct., vol. 79, pp. 1097-1107, 2001. 
[29] A. Kurtz, Y. Tang and F. Zhao, "Intra|Lattice: Generative Lattice Design with Grasshopper," 2015. [Online]. Available: http://intralattice.com.

[30] S. Antman, Nonlinear Problems of Elasticity, vol. 107, New York: Springer, 2005.

[31] T. J. R. Hughes, J. A. Cottrell and Y. Bazilevs, "Isogeometric analysis: CAD, finite elements, NURBS, exact geometry and mesh refinement," Comput. Methods Appl. Mech. Eng., vol. 194, no. 39-41, 2005.

[32] O. Weeger, B. Narayanan, L. De Lorenzis, J. Kiendl and M. Dunn, "An isogeometric collocation method for frictionless contact of Cosserat rods," Comput. Methods Appl. Mech. Eng., vol. 321, pp. 361-382, 2017.

[33] "PolyJet Materials Data Sheet," Stratasys Ltd., [Online]. Available:

http://global72.stratasys.com/ /media/Main/Files/Material_Spec_Sheets/MSS_PJ_PJMaterialsDataSheet_0517aW eb.pdf. [Accessed 28 Aug 2017]. 